\title{
Comparative study to evaluate the success rate of manual vaccum aspiration and Medical abortion in termination the first trimester pregnancy
}

\author{
Bhardwaj $M^{1}$, Bhargava $S^{2}$ \\ ${ }^{1}$ Dr Malini Bhardwaj, Associate Professor, Department of Obstetrics \& Gynecology, RKDF Medical College, Bhopal, \\ MP, India, ${ }^{2}$ Dr Sumit Bhargava, Associate Professor, Department of Anesthesiology, L.N. Medical College Bhopal, MP, \\ India
}

Address for correspondence: Dr Malini Bhardwaj, Email: dr.malinibharadwaj@gmail.com

\begin{abstract}
Objectives: This study was undertaken to evaluate the success rate of manual vaccume aspiration in termination the first trimester pregnancy, study the merits and demerits of the method and compare it with other method i.e. medical abortion by misoprostol and mifepristone in first trimester MTP. Methodology: In a prospective randomized controlled study, participants were divided in two groups: Study group of 30 patients of 6-12 weeks gestational age subjected to MTP by manual vacuum aspiration. Another group consisted of 30 patients of 6-8- weeks gestational age who were subjected to MTP by medical method using mifepristone and misoprostol. Result: Present study consist of 30 cases of medical termination of pregnancy in $\mathrm{I}^{\text {st }}$ trimester carried out by (manual vaccum aspiration) MVA. A comparison of observation and results was made with another group of 30 patients who had undergone pregnancy termination in $\mathrm{I}^{\text {st }}$ trimester using mifepristone and misoprostol. In 6-8 weeks pregnancies incidence of complete abortion was $100 \%$ In 10 weeks complete termination was done in 8 patient's only 1 patient had incomplete termination. In medical abortion also results show that in up to 6 week pregnancy 3(12\%) patient had incomplete expulsion, in up to 8 week 11(20\%) patient had incomplete expulsion In present study over all success rate with MVA was 93.33\% whereas with medical abortion success rate is found to be $86.67 \%$. Conclusion: It is concluded from present study that Manual Vaccum Aspiration is safe, simple and easy procedure with minimal need of anesthesia and analgesia. It is very good option in low resource settings.
\end{abstract}

Keywords: Manual Vaccum Aspiration, Medical Termination of Pregnancy, Medical Abortion

\section{Introduction}

Fertility control through induced abortion though an ancient practice, continues to be popular in present day obstetrics as well. Contraception is definitely a far superior way of family and population limitation than termination of pregnancy. Yet it cannot be ignored that the lack of fore sight often leaves an individual with burden of an unwanted or illegal pregnancy. Number of such pregnancies is significantly enough to render pregnancy termination an important method of fertility control.

Before the era of legalized abortion, such pregnancy termination was considered illegal by law, therefore it was domain of untrained quacks who performed them

Manuscript received: $4^{\text {th }}$ Aug 2015

Reviewed: $10^{\text {th }}$ Aug 2015

Author Corrected: $17^{\text {th }}$ Aug 2015

Accepted for Publication: $30^{\text {th }}$ Aug 2015 most unhygienically which made significant contribution to maternal morbidity \& mortality rates.

Intense realization about the grave consequences of population explosion all over the world led to formation of abortion laws in various counties. The pressing need of time made the policymakers to revise the law of abortion thereby leading to liberalization of indications for abortion.

In India, the bill on medical termination of pregnancy was passed through parliament in 1971 and brought into execution on April 1972[1]. This revolutionized the sphere of obstetrics, which deals with pregnancy termination and also made tremendous impact on religious and cultural values of present day social setup. 


\begin{abstract}
Abortion has been legal in India since 1971, The law is quite liberal, as it aims to reduce illegal abortion and maternal mortality. An abortion can be performed in India until the 20th week of pregnancy. The opinion of a second doctor is required if the pregnancy is past its 12th week. The Medical Termination of Pregnancy Act was amended in 2002 and 2003 to allow doctors to provide mifepristone and misoprostol (also known as the "medical abortionl") on prescription up to the seventh week of pregnancy. With legalization of abortion, demand for better, more reliable and safer method of pregnancy termination in first trimester has become more pressing. Safety of pregnancy termination is inversely related to the gestational period. First trimester abortions are relatively easy to perform and are attended by fewer complications and unpleasant sequels, whereas mid trimester abortion mortality rates are likely to be 3 to 4 times greater than those associated with first trimester pregnancy terminations. There are various method of first trimester pregnancy termination like mifepristone (RU-486) misoprostal ePGE2 (Gameprost) in medical methods and menstrual regulation, manual vacuum aspiration and suction evacuation in surgical methods. But the search for safer solution led to discovery of MVA. This study was conducted to evaluate the safety and effectiveness of Manual Vacuum Aspiration in first trimester MTP.
\end{abstract}

\section{Methodology}

Design: Prospective comparative study,

Setting: Department of Obstetrics and Gynecology in GRMC Gwalior and Associated, Hospital over a period of 18 months.

Selection of cases: participants for study were selected in two groups:

Sample size: Study group of 30 patients of 6-12 weeks gestational age subjected to MTP by manual vacuum aspiration. Another group consisted of 30 patients of 68- weeks gestational age who were subjected to MTP by medical method using mifepristone and misoprostol.

Inclusion criteria: Patients of early pregnancy (6-12 weeks in MVA group and 6-8 weeks in medical abortion) willing for termination of pregnancy were included in the study.

\section{Exclusion criteria}

1- Suspicion of ectopic pregnancy

2- Pregnancy with fibroid uterus.

3- History of caesarean section or uterine surgery

4- Severe cervical stenosis

5- Pelvic inflammatory disease.

6- Medical disorders anaemia, renal diseases, heart diseases, bleeding disorder.

\section{Material}

MVA syringe is a $60 \mathrm{cc}$ syringe (Usually made with polyethylene) with a capacity to hold vacuum of 25-26 inches of $\mathrm{Hg}$ which is equivalent to the vacuum created in an electric suction pump, sterile gloves, sim's or Cusco's speculum, anterior vaginal wall retractor, allis forceps (long)/volsellum (small toothed),sponge holding forceps.

For medical method tablet Mifepristone and tablet Misoprostol are required. Mifepristone is a substituted 19 non steroid compound clinically designated as $11 \beta$ [p-dimethylamine pheny1] 17 hydroxy 17- (1propyny1) octra 4,9 dien 3 one. It acts by binding to human progesterone receptors. Misoprostol is a prostaglandin

\section{Procedure}

\section{MVA technique/procedure}

\section{Preparation of vacuum syringe and canulae}

i- Close the pinch valve by pushing the buttons down forward towards the syringe tip (can feel the valve locks)

ii- Pull back on the plunger until the arms of the plunger snap outward at the end of the syringe barrel holding the plunger in place.

iii- Check the stable positioning the plunger arms (the plunger arms must be fully extended to the sides and secured over the edge of the barrel). With the arms in this position, the plunger will not move forward and vacuum is maintained.

2. The procedure: Patients is put in lithotomy position after emptying the bladder. After part preparation and $\mathrm{P} / \mathrm{v}$ examination hold the anterior lip of the cervix using volsellum.

i- Insert the canulae through the cervix into the uterine cavity just past the internal os by rotating the canulae while gently applying pressure. Start with canula of size 4 and gradually increase to the size that corresponds with the period of gestation.

ii- Push the chosen canula slowly into the uterine cavity just beyond the os until it touches the 
fundus. Note the uterine depth by the dots visible on the canula (the dot nearest to the tip of the canula is $6 \mathrm{~cm}$ from the tip and each dots are at 1 cm interval.)

iii- After measuring the uterine depth, withdraw the canula slightly.

iv- Attach the prepared syringe to the canula holding the Allis forceps and the end of the canula is one hand and the syringe in the other ensuring that the canula does not move forward.

$\mathrm{v}$ - Release the pinch valve on the syringe to release the vacuum into the uterine cavity. Tissue and bubbles should begin to flow through the canula into the syringe.

Complications like excessive bleeding, incomplete evacuation were noted.

\section{In patients care after the procedure}

i- Check the vital signs while Patients is still on operation table.

ii- Check for bleeding per vagina and vital signs.

iii- Discharge the patients when she is stable (usually after two hours).

iv- Oral methyl ergometrine, analgesic and antibiotic are given after discharge.

\section{Results}

Present study consists of 30 cases of medical termination of pregnancy in Ist trimester carried out by MVA in department of Obst. \& Gynae. GRMC, Gwalior. A comparison of observation and results was made with another group of 30 patients who had undergone pregnancy termination in Ist trimester using mifepristone and misoprostol during January 2002 to July 2003.

Table No 1: Shows comparable age incidence (cumulative) of patient in MVA and medical abortion series

\begin{tabular}{|l|l|l|l|l|}
\hline Age in yrs & MVA & \multicolumn{2}{l|}{ Medical abortion } \\
\hline & No. of cases & $\%$ & No. of cases & $\%$ \\
\hline Up to 20 & 0 & $0 \%$ & 0 & $0 \%$ \\
\hline $21-25$ & 6 & $20 \%$ & 14 & $46.67 \%$ \\
\hline $26-30$ & 18 & $60 \%$ & 12 & $40.00 \%$ \\
\hline $31-35$ & 2 & $6.67 \%$ & 4 & $13.33 \%$ \\
\hline $36-40$ & 3 & $10 \%$ & 0 & $0 \%$ \\
\hline$>40$ & 1 & $3.33 \%$ & 0 & $0 \%$ \\
\hline
\end{tabular}

Mean age in MVA group - 29.3 years

Mean age in medical abortion group -26.66 years.

Table No 2: Shows time taken for the procedure at different gestational age.

\begin{tabular}{|l|l|l|}
\hline Period of gestation & No. of patients & Mean Time taken \\
\hline 6 weeks & 8 & $10 \mathrm{~min}$ \\
\hline 8 weeks & 12 & $15 \mathrm{~min}$ \\
\hline 10 weeks & 9 & $18.8 \mathrm{~min}$ \\
\hline 12 weeks & 1 & $25 \mathrm{~min}$ \\
\hline
\end{tabular}
given on day one, followed by two tablet misoprostol (400 mcg) orally after $48 \mathrm{hrs}$ and again two tablets misoprostol $400 \mathrm{mcg}$ after $12 \mathrm{hrs}$. Observations were made about pattern of bleeding, abdominal cramps, any other side effects and see whether the expulsion is complete or not.

Statistical Analysis: Statistical analysis was done with Stata 11 software. Demographic characteristics, marital status, parity, age, educational status and complications were compared between two groups and data was analyzed statistically. for continuous variables descriptive statistics(mean and standard deviations) were computed. Comparison of means in MVA group and medical abortion group was done using unpaired $\mathrm{t}-$ test. For categorical data chi-square test was applied. $\mathrm{P}<0.05$ was considered significant.

v- Patients were asked to contact the doctor immediately if any of the following happens:

- Bleeding more than menstrual flow

- Severe or increased pain

- Prolonged bleeding (more than two weeks)

- Foul smelling discharge per vagina 
Mean time taken in 30 patients $-14.47 \mathrm{~min}$. The mean time taken for the procedure increase with gestational age.

Table No 3: Shows relation of complete and incomplete termination with gestational age in MVA series.

\begin{tabular}{|l|l|l|l|}
\hline Weeks in gestation & No. of cases & Complete & Incomplete \\
\hline 6 weeks & 8 & 8 & - \\
\hline 8 weeks & 12 & 12 & - \\
\hline 10 weeks & 9 & 8 & 1 \\
\hline 12 weeks & 1 & 0 & 1 \\
\hline
\end{tabular}

As is evident from above table the success rate decreases with increasing gestational age after 10 week this method is infective in our study.

Table No 4: Shows associated side effect in MVA series.

\begin{tabular}{|l|l|}
\hline Side effect & No. of patients \\
\hline Excessive bleeding & 1 \\
\hline Perforation & - \\
\hline Incomplete evacuation & 2 \\
\hline Pain during procedure & 7 \\
\hline
\end{tabular}

Complications may occur in MVA as seen in above table.

Table No 5: Comparative study in MVA and medical abortion in 6-8 week gestation.

\begin{tabular}{|l|l|l|l|l|}
\hline \multirow{2}{*}{ Side effect } & MVA & \multicolumn{3}{l|}{ Medical abortion } \\
\cline { 2 - 5 } & 6 week & 8 week & 6 week & 8 week \\
\hline No. of cases & 8 & 12 & 25 & 5 \\
\hline Complete termination & 8 & 12 & 22 & 4 \\
\hline Incomplete & 0 & 0 & 2 & 1 \\
\hline Failed & 0 & 0 & 1 & 0 \\
\hline
\end{tabular}

It is obvious from above table that success rate in MVA group is $100 \%$ in upto 8 weeks gestation while it is $88 \%$ in 6 weeks and $80 \%$ in 8 weeks gestation. Failure rate or rate of incomplete abortion is $0 \%$ in MVA group while in medical abortion group rate of incomplete expulsion is $8 \%$ in 6 weeks and $20 \%$ in 8 weeks. Failure rate was $4 \%$ in 6 weeks in medical abortion group.

$\mathrm{P}$ value of complete termination in 6 and 8 weeks gestation is 0.003 and 0.027 respectively and $\mathrm{P}$ value of incomplete termination of incomplete termination in $6 \& 8$ weeks is $0.015 \& 0.027$ respectively. Thus it is concluded that this difference is significant.

Table No 6: Comparative study in MVA and medical abortion group up to 8 week gestation.

\begin{tabular}{|l|l|l|}
\hline Side effect & MVA & Medical abortion \\
\hline No. of cases & 20 & 30 \\
\hline Complete & 20 & 26 \\
\hline Incomplete termination & 0 & 3 \\
\hline Failed & 0 & 1 \\
\hline Excessive bleeding & 0 & 4 \\
\hline Pain & 6 & 6 \\
\hline
\end{tabular}




\section{Discussion}

In India, the bill on medical termination of pregnancy was passed through parliament in 1971 and brought into execution on April 1972[1]. This revolutionized the sphere of obstetrics, which deals with pregnancy termination and also made tremendous impact on religious and cultural values of present day social setup. Abortion has been legal in India since 1971, when the Medical Termination of Pregnancy Act was passed. The law is quite liberal, as it aims to reduce illegal abortion and maternal mortality. An abortion can be performed in India until the 20th week of pregnancy. The opinion of a second doctor is required if the pregnancy is past its 12 th week. Late pregnancy termination caries more risk then early pregnancy termination and this has led to discovery of a variety of methods which will render the early pregnancy termination more safe and effective with minimum side. Manual vacuum aspiration is one of them.

Surgical methods especially vacuum aspiration in early pregnancy is safe, reliable and quick and yet there is a definite place for medical method for termination of pregnancy. There are many women who prefer to avoid surgery and want secrecy.

In the present study an attempt was made to evaluate the usefulness of MVA as a method of trimester pregnancy termination. A relative evaluation of MVA and medical abortion was done by comparing the observation and results of patients in MVA series with those of similar group of patients in whom pregnancies were terminated by medical method at almost the same gestational age as that of MVA. This comparison was made considering age, parity, gestation age of pregnancy and efficacy, side effects and complication etc.

There is fear of surgery specially when adolescent girls have unwanted pregnancy and need help. The effectiveness of medical abortion has been well demonstrated in a series of trial by Krishna and Coyaji undertaken in variety of settings in India [2]. Success rate is very good (98\%) up to 50 days of LMP but this decline to some extent when the period of amenorrhea extends. Side effects are nausea, occasional vomiting and abdominal cramps. Occasionally there may be mild diarrhea.

I M Spitz et al reported an incidence of 11.6 per abortion 1000 live birth before legalization of abortion in year 1967. [3] After which the incidence has increased 8 times the previous one.

Age: In the present study patients who sought MTP were from 22 to $42 \mathrm{yrs}$. of age. Incidence was maximum in 26-30 yrs of age group (60\%) Incidence declined sharply after 35 yrs. In 35-40 yrs age group it was minimum as fertility also to fall after the age of 35 yrs. In medical abortion groups was not very much different i.e. 29.3 yrs. in MVA and 26.66 in medical groups.

Gestational Age : In the present study $8(26.67 \%)$ of all patients who were aborted with MVA had 6 weeks size uterus $12(40 \%)$ had 8 weeks uterus and $9(30 \%)$ of patient had 10 weeks uterus $1(3.33 \%)$ had 12 wk size uterus. FOGSI Ipas (2007) conducted multicentric study in settings of medical college hospitals to private hospitals and clinics of India [4]. In which 926 patients were taken, out of them $39.8 \%$ performed prior to $6^{\text {th }}$ week $33.8 \%$. In medical abortion series $25(83.33 \%)$ patient had up to 6 weeks at $5(16.6 \%)$ of patients had up to 8 weeks.

\section{Complication}

Incomplete Abortions: In MVA series percentage of patients of complete abortion decreased with increased gestational age. Only 2 patients had on incomplete evacuation. They needed electric suction and curettage. In 6-8 weeks pregnancies incidence of complete abortion was $100 \%$. In 10 weeks complete termination was done in 8 patient's only $1(11.11 \%)$ patients had incomplete termination. In 12 weeks group all patient had incomplete abortion. The above results show that definite improvement in quality of abortion with decreased gestational age. In MVA series termination is quick, success rate is higher and evacuation more complete with decreased gestational age as compared to increased week of pregnancy.

In medical abortion also results show that in up to 6 week pregnancy $3(12 \%)$ patient had incomplete expulsion, in up to 8 week $11(20 \%)$ patient had incomplete expulsion. Hemlin J, Mollor B, (2001) study done in Sweden including 170 patients who were randomized in VA and MVA, 88 and 91 patients respectively[5]. Two patients in each group has incomplete evacuation.

Excessive bleeding: In our study one patient at 10 weeks gestation had excessive bleeding which was 
managed without any further complication. Das Vinita and Jain Swati studied cases and found that incidence of blood loss was 50\% lower with MVA than sharp curettage [6]. In one study pattern and predictors of bleeding after early abortion with mifeprstone and misoprostol or MVA were determined [7]. 212 Women were enrolled in which $80 \%$ women completed the study. Mean days of bleeding were higher in medical (14 days) than MVA (9 days) group. In medical abortion group 4 patients $(13.33 \%)$ had excessive bleeding.

Incidence of side effects: There was pain during MVA in $7(23.33 \%)$ cases out of 30 cases. In medical abortion group $6(20 \%)$ patients had abdominal pain and camps. Side effects like nausea, vomiting, diarrhea were not reported in our study. [8]

Duration of Hospital Stay: Mean duration of hospitals stay in present study was $5.9 \mathrm{hr}$. In medical abortion patients can be given drugs in outdoor setup. There is no need of hospitalization. In Hemlin $\mathrm{J}$, Mollor B, (2001) study including 200 patients $(80 \%$ completed the study) mean duration of hospital stay was $2 \mathrm{hr}$.[5]

Failure Rate: Over All Failure rate with MVA was $6.67 \%$ and with medical abortion was $13.33 \%$. Hemlin J, Mollor B (2001) study done in Sweden included 179 patients[5]. They were randomized in VA and MVA group (91 in MVA \& 88 in VA group). There was no significant difference in frequency of complete abortion. Two patients in each group subsequently needed re-curettage because incomplete evacuation.

Success Rate/ Effectiveness: In present study over all success rate with MVA was $93.33 \%$ whereas with medical abortion success rate is found to be $86.67 \%$. Given the potential of MVA in allowing simplification of requirement for safe abortion service as suggested by the evidence presented earlier it is today our best hope in promoting widespread, nationwide decentralized safe abortion services through the governmental WHO and private sectors. In keeping traditions of medicine, MVA is a relatively low cost, low tech procedure with few logistic demands that has the potential to decentralize and democratize safe legal abortion services in India, contribute to the cause of safe motherhood and ensure every enemy couple the right and an option to cope with unwanted pregnancy with safe and dignity.

\section{Conclusion}

It is concluded from present study that MVA is a very simple, safe and easy procedure. It is very convenient and free from any side effects. It does not require any special skill for it. Need of anesthesia and analgesia is also minimal. It is very effective in setups where minimal facilities are available. But with these advantages it has some disadvantage like patients need hospitalization for the procedure, which is not necessary in medical method.

\section{Funding: Nil \\ Conflict of interest: None. \\ Permission of IRB: Yes}

\section{References}

1. Yadav V K, Sharma P : .Medical termination of pregnancy-An overview,Ind $\mathbf{J}$ Pharma and Bio Science2013 Apr;(p)740-744.

2. Coyaji K, Elul B, Krishna U, Otiv $S$ et al. Mifepristone-misoprostol abortion: a trial in rural and urban Maharashtra, India. Contraception. 2002 Jul;66(1):33-40.

3. Spitz $\mathrm{IM}^{1}$, Bardin CW, Benton L, Robbins A. Early Pregnancy termination with mifepnistone \& misoprostol in the united states. N Engl J Med. 1998 Apr 30;338(18):1241-7.

4. FOGSI IPAS 2007 J Obstet Gynecol India, 57(2):162

5. Hemlin, Johan and Bo Moller. 2001. Manual vaccum aspiration, a safe and effective alternative in early pregnancy termination. Acta Obstet Gynaecol Scand, 80:563-567.

6. Das V, Jain S, Gupta Hem Prabha et al. Evaluation of newer methods of early pregnancy termination. I obstet gynaecol India 55,no.5;September/October 2005 pg 454-456.

7. Davis A, Westhoff C, De Nonno L. Bleeding patterns after early abortion with mifepristone and misoprostol or manual vacuum aspiration. J Am Med Womens Assoc. 2000;55(3 Suppl):141-4.

8. Hakim-Elahi E, Tovell HM, Burnhill MS. Complications of first-trimester abortion: a report of 170,000 cases.

Obstet Gynecol. 1990 Jul;76(1):129-35. 


\section{How to cite this article?}

Bhardwaj M, Bhargava S. Comparative study to evaluate the success rate of manual vaccum aspiration and Medical abortion in termination the first trimester pregnancy. Int $J$ Med Res Rev 2015;3(8):884-890. doi: 10.17511/ijmrr.2015.i8.166. 